\title{
Evoking the Post-industrial Landscape Memories through Spectrality and Mixed Reality Soundscapes
}

\author{
Yixiao $\mathrm{Fu}$ \\ yx941104@hotmail.com \\ School of Architecture, Carnegie \\ Mellon University \\ Pittsburgh, Pennsylvania, USA
}

\author{
Daragh Byrne \\ daraghb@andrew.cmu.edu \\ School of Architecture, Carnegie \\ Mellon University \\ Pittsburgh, Pennsylvania, USA
}

\author{
Lawrence Shea \\ 1shea@andrew.cmu.edu \\ School of Drama, Carnegie Mellon \\ University \\ Pittsburgh, Pennsylvania, USA
}

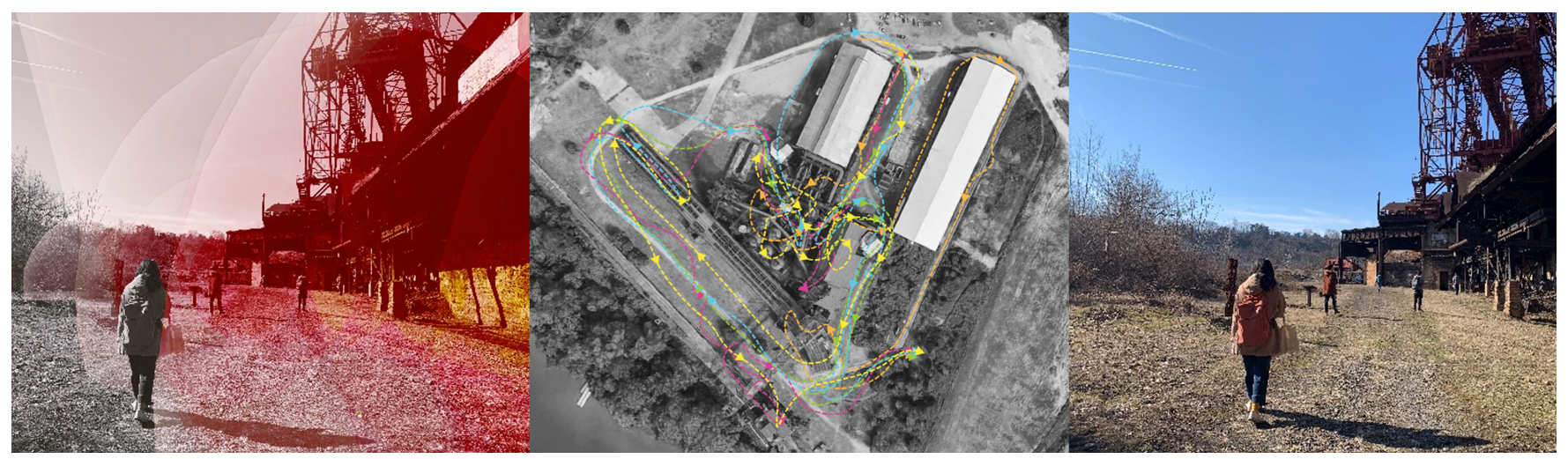

Figure 1: Ïnner Memory of the Post-Industry; a site-specific mixed reality audio walk at Carrie Furnace

\begin{abstract}
Spectrality describes the spatiotemporal relationships between landscape and memories where the past 'haunt' the present. We explore how spatialized soundscapes may evoke the spectral memories of physical landscape. To do this, we curated a site-specific mixed reality audio walk at Carrie Furnace, a national historic landmark in Pittsburgh's 'Rivers of Steel' National Heritage area. This audio walk leverages virtual soundscapes, mobile mixed reality, and openended narratives. Each visitor takes a unique path through the spectral memories of the site's industrial past and post-industrial relics. Findings from an eleven-person user study assess our approach to spatialized spectral soundscapes and reveal the dimensions (content, context, experience) that resonate with audiences.
\end{abstract}

\section{CCS CONCEPTS}

- Applied computing $\rightarrow$ Sound and music computing; $\bullet$ Humancentered computing $\rightarrow$ Mixed / augmented reality.

\section{KEYWORDS}

Spectrality, Audio-based Mixed Reality, Soundscape, Post-Industrial Landscape, Audio walk

\section{c) (7) (8)}

This work is licensed under a Creative Commons Attribution-NonCommercial International 4.0 License.

C\&C '21, fune 22-23, 2021, Virtual Event, Italy (c) 2021 Copyright held by the owner/author(s). ACM ISBN 978-1-4503-8376-9/21/06.

https://doi.org/10.1145/3450741.3465256

\section{ACM Reference Format:}

Yixiao Fu, Daragh Byrne, and Lawrence Shea. 2021. Evoking the Postindustrial Landscape Memories through Spectrality and Mixed Reality Soundscapes. In Creativity and Cognition (C\&C'21), June 22-23, 2021, Virtual Event, Italy. ACM, New York, NY, USA, 6 pages. https://doi.org/10.1145/ 3450741.3465256

\section{INTRODUCTION}

Spectrality was first introduced by Jacques Derrida [16]. He used this term to describe the temporal and ontological disjunction where the "living present" is "out of joint." Joseph describes, of Derrida's hauntological approach, how this difference between the present and the past meanings that "always leaves behind a ghostly trace [24]," or in Derrida's own words: "a simulacrum of a presence that dislocates itself, displaces itself [15]." It promotes consideration of the spatiotemporal relationships between a place, the self, and its spectral memories; 'haunted' encounters occur where the past and present co-exist and the past can be temporarily perceived and interact with the present [15, 24, 45]. Wylie notes that author W.G. Sebald enlivens this concept through his "sustained meditations upon relationships between place, memory and subjectivity" in notable works such as 'The Rings of Saturn' [45]. Sebald's work centers on wandering voyages of richly described landscapes, "characterized by irruptions of the surreal and the phantasmagorical," often to unsettle, trouble and complicate the landscapes being explored [45]. Spectrality and spectral memories, as such, provide vehicle for enacting a disjointed temporality around a place, and suggest an unconventional way to understand and experience contemporary landscapes and their histories. We take inspiration from Sebald and Derrida in the construction of a new audio-based mixed reality (MR) 
experience of the landscape. We use sound as a physically unconstrained and invisible medium, ideally suited to crafting a spectral presence for a landscape's past. We do this in the context of historically and culturally unique spaces like post-industrial landscapes. Prior work by Latz (as discussed in [8]) emphasizes the importance of preserving existing structure while adding the new materiality to catalyze metamorphosis of the post-industrial landscape. Latz's concept focuses on the visuality, materiality, and physicality of the site, we instead focus on sound. Sound is a powerful tool to weave together the sensorial experience of place and it also avoids interfering with the condition of the site. Additionally, and from an experiential frame, we regard site-specific sound design as an opportunity to add a new spectral materiality to the landscape.

Located at the interface between sound, spectral memory, postindustrial landscape, and contemporary media technology, this work explores how spatialized soundscapes may evoke the spectral memories of physical landscape. We discuss the design, development, and deployment of an hour-long fully-spatialized MR audio-walk experience, "Inner Memory of the Post-Industry" at Carrie Furnace, a Pittsburgh historical landmark. This site-specific immersive spectral soundscape reveals the experience of working in a blast furnace and encounters with oral history from Carrie Furnace's past. Our approach uses an open-ended, unguided, spacedriven auditory wandering inspired by Sebald. An evaluation with 11 participants describes how the spatialized soundscape complicated the present condition of the site, created a sense of spectrality, and allowed participants to experience temporal disjoints as they toured the site.

\section{BACKGROUND}

Spectrality has been explored primarily in the humanities to examine the "spatial sedimentation of history and tradition," "intricacies of memory and trauma," and "technology and media" [6]. It has been applied in cultural geography research [31] and in tours of heritage sites [30]. While Gatehouse recently used hauntology in participatory design to re-presence past encounters with hate [19], it remains under-explored in HCI. Yet concepts of landscape and memory that are embedded in spectrality have value in experience design for cultural heritage [44]. While some work, such as 'Jewish Time Jump' [20] recognizes this, these exploration prioritize visual interventions and game-based strategies [22, 44]. There is however much prior work in curating site-specific soundtracks as a way of experiencing specific places. Audio tours have been widely used in museum and cultural heritage contexts to enhance education, public engagement, and the interactivity of exhibitions $[29,42]$. One notable example is the immersive walking tour of the Alcatraz Cellhouse, created by Chris Hardman in 1987. Visitors experience interviews from inmates, guards and other stories in tandem with evocative ambient sounds as they walk through the prison. This experience is "clearly focused on moving from a traditional interpreter-led format to one with a direct emotional impact, achieved by making guard and inmate voices actually present." [7]. Audio walks are a form of artistic practice in their own right [9]. The term "audio walk" originates from works by Butler and Cardiff $[10,11]$. They offer "very powerful presentations linking spoken memory with specific places [9]." This is best exemplified by
Janet Cardiff's 'Jena Walk', a site-specific audio walk centered on a battle between the Prussians and Napoleon's army [11]. Listeners encounter 'time slips' - sound elements from various time periods are intentionally mixed - making them aware that the landscape is shared with others throughout history. This piece is directly related to notions of spectrality. The role of walking in audio tours is also widely discussed: walking helps to "illuminate the relationship between space, time, and the body [21]" and its temporality, rhythmicity, and variable duration is a resource for, if not a form of, artistic practice itself $[21,35]$. These considerations are found in audiobased artworks such as Remote X [38], Blast Theory's "Too much information," [41], and [murmur] [18]. Ciolfi and Petrelli [13] use walking a tool to reveal the multilayered values in cultural heritage sites. This draws on the ethnographic field walks of Ingold and Vergunst [23]. Interactive performance research [4] is often organized around explorations of locative media. "Place-based sound" couples sound with location metadata by using GPS or indoor positioning to activate playback by walking to points of interest, allowing people to explore soundscapes layered on physical spaces. Place-based audio traditionally falls into two primary strategies. The first is user-driven which gives interactive control to the visitor to select, filter, identify and/or control content playback. This agency places effort on the audience to effect their experience and may distract them from the subject matter. Alternatively, Benford et al. suggest trajectories - a journey designed using hybrid structures of time, space, interaction, and roles - to craft continuity and coherence in wandering, open-ended exploration, and non-linear encounters $[3,12]$. We believe that an open-ended, trajectory-based approach is essential to realizing spectrality in place-based audio. This work also relies on interactive techniques for MR sound-experiences and for immersive audio tours. Prior work includes: Sotto Voce's mechanisms for 'audio eavesdropping' [1]; ec(h)o's playful approaches and tangible interfaces for soundscapes [43]; Eyes Free Art's proxemic audio interactions [39]; Ghosts in the Garden's [36] 'chooseyour-own-adventure' game; Behrendt's experiments with remixing place-based sound through the act of walking [2]; and Dow et al's use of spatial narratives to structure experiences in mixed reality audio tours [17]. Most related to our work is Spomenik's audio tour of Slovenia's Stalinist purges and its examination of spatial memory and relational geography in the design of networked monuments [25]. Despite offering rich interactive strategies [28], audio-based MR rarely takes full advantage of spatial approaches, e.g fully-spatialized audio or activation through contextual and behavioral cues. This stems from the many logistical, technical, and narrative challenges in authoring location-based experiences [12]. Further, performance-based research is challenged by "complex interrelationships between artistic-practice, studies and theory" [4]. As such, there are many emerging interactive strategies that are under-explored in MR soundscapes.

\section{DESIGN AND IMPLEMENTATION}

Site and Context: Pittsburgh once produced more than $60 \%$ of the total output of steel for the US. The Carrie Blast Furnace was built in 1881. The structure of Carrie Furnaces 6 and 7 have remained virtually unchanged even after the modernization of structure in 1936 (see Figure 2) [34]. They were the first furnaces on the site to be 


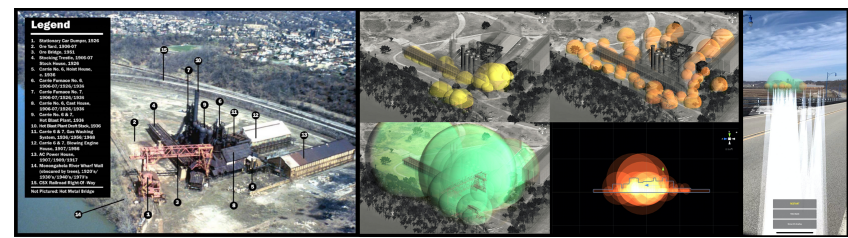

Figure 2: Left: Carrie Furnace Structure Map. Middle: Soundscape Visualization in Unity (Yellow: individual sound effects for specific structures, Orange: human voice clip, Green: looping ambient sound effects) Right: System Interface

shut down, but this also made them suitable, and ultimately chosen for preservation. In 2006, Carrie Furnace became a 35 acre National Historic Landmark, now managed by the local non-profit Rivers of Steel $[32,33]$. There are 15 major structures and areas at the site (Figure 2). Visitors primarily experience these externally; however, many interior spaces are accessible. The site also includes several art works, sculptures, and graffiti walls. Highlights include the Carrie Deer, a sculpture prepared by artists operating on the sites, the stationary car dumper, a structure for transporting raw materials, and the stocking house, one of the most severe workspaces at the site. Actively touring these spaces takes between 15 to 30 minutes.

Our audio walk is organized around eleven of these structures. It was orchestrated as a MR mobile application that plays back fully spatialized media based on the position, orientation, and proximity to spatiotemporal audio targets. Spatialization is leveraged for richer sense of the audio content being embedded in the environment. The virtual audio behaves as if placed in real space: as you approach it becomes louder, and as you turn, it may be more discernible by one ear versus the other. Spatialization and the experience of encountering virtual media in the environment it creates was a key feature in realizing spectrality in this work. The parameters for triggering sound were tied to a virtual 3D 'volume' that changed shape and moved over time to increase serendipity, encourage openended exploration and further a sense of spectrality for visitors. Our effort was organized into five key processes, described below.

Content Curation: Audio content about the history and impact of Carrie Furnace was first gathered, edited, and assembled. This was of two primary types: atmospheric sounds and oral histories. A library of environmental sound was developed from sources including the BBC sound effects [14] and the U.S. Steel corporation's sound collections. Oral histories consisted of interviews with former workers and artists gathered from the Rivers of Steel oral history archive and open-source documentaries. The raw content was coded into themes, sub-themes, and topics to assist with planning the narrative soundscape (e.g. workplace activities, safety concerns, artistic activities.) After analysis and coding, clips were further edited for duration, and curated, labelled, and organized into a cue sheet.

Soundscape Composition: We conducted this phase in a simulated virtual model of Carrie Furnace where spatial relationships could be easily and iteratively evaluated to form the final soundscape (see Figure 2.) This was prepared in Unity to create experience simulations and later to coordinate sound with the physical site. The virtual replica of the site provides an accurate reference for both the structural and spatial relationships with the real environment that influence the design of the digital contents in the MR experience. It enabled the designer to quickly experiment with arranging audio elements at various locations and at scale. Constructing narrative sequences based on landscape differ from those in other mediums as the participant moves through the medium itself at his/her chosen speed while potentially engaging in various activities [37]. Based on both the audio content and spatiality of the site, we used multiple complimentary strategies, including attaching virtual audio to directly related physical structure forming an atmospheric contrast, adding interest to open fields, and spatially grouping related subtopics. This last strategy was found to be particularly important; a scattered composition creates a disjointed experience and eliminates a sense of a suggested circulation. For example, the stocking house, one of the harshest working environments on the site, has an eerie quality owing to the fact that both the sonic and visual experience inside it is drastically different from any other place on the site. To accentuate this, our soundscape introduces archival sounds of the work that took place here, including steel material dropping and the rail car moving. This content is placed in a way so that people hear a chain of sound effects aligned with original systems. Alternative versions of the audio with applied reverberation effects was leveraged to emphasize the cavernous, echoing environment. Due to the unsettling, gloomy sensibility of this area, many visitors were reluctant to go inside. We also designed virtual sound objects with moving paths to leads them in.

Interaction Design: Much prior work on performance, interactivity and immersion informed the design of our experience [2, 3, 5, 40]. By incorporating support for a fully embodied, three-dimensional and immersive audio playback, the 3D relationship between the listener, sound, and their situated environment affords the virtual soundscape other opportunities, including independent movement of each sound object. The spatialized sounds adapt to their spatialized presence in the environment [26], which helps to emphasize sound as individual spectral memory. Unlike traditional audio walks and tours which break the walking experience at each static moment of content, this approach reinforces a continued walking rhythm and promotes non-linear and open-ended exploration [3]. As an immersive experience, presence does not only come from the environment but from the participant's sense of "being there". Rather than actively controlling the auditory experience, the participant's spectatorship during the walk is what drives the interaction; this is leveraged to better convey the presence of spectral memory. During the tour, sound objects will start to "wander" within the environment, independent of the visitor's movement. The initial speed of each sound object (except for ambient sounds) is randomly initialized to be between 0.5 to 1.5 feet per second to mimic normal walking speed. When a sound object is encountered by visitor, it will play and slow down for visitors to explore. Visitors are free to move away from the triggered sound at any time, but each sound can only be triggered once. This fleeting aspect of the media further reinforces spectrality, serendipity and open-ended exploration.

Mobile Application Development: A mobile application was built for the final on-site experience. Prototyping on-site allowed the mapping between the virtual objects to specific geo-locative and 
contextual triggers to be tested. It also served to evaluate the performance of spatialized virtual sounds against the real environmental acoustic experience. The initial mobile prototype was built in Unity, and used Unity AR Foundation SDK and Unity AR+GPS Location assets. The tracking system is enabled by smartphones' GPS and built-in magnetometer gyroscopic sensor. Although the final experience is sound-based, screen-based interface is still available for debugging and monitoring. The prototype was tested on-site with location markers and sound examples which were given various spatialization settings. Prototype field testing validated that marker objects were successfully placed and detected.

\section{EVALUATION AND FINDINGS}

To evaluate the design, eleven participants (four women, seven men) were recruited participants through mailing lists and known networks and invited to the Carrie Furnace at the same time and date. Participants were between 20 and 30 years of age and were required to be fluent in English. The MR app was installed to the participants' personal devices prior to arrival. They completed the on-site audio walk (for 75 to $90 \mathrm{mins}$ ), followed by an on-site focus group discussion (30 mins.) Transcripts were analyzed using bottomup iterative coding. Findings are next discussed.

Perception of Spectrality: The focus of this project is to use of sound to present the spectral presence of a landscape's memory, legacy, and history. Presence occurs when people can perceive a medium's content (i.e. objects or entities) without noticing the medium itself [27]. P8 noted this began from the earliest moments of the audio tour: "the strong sounds of machines and people came in and I feel that the past and the present are mixed..." This blurring of past and present is essential for spectrality to be felt. As participants began to perceive and interact with the past through oral histories and ambient audio, this disjointed temporality was further revealed. P10 mentioned that encountering different moving sounds gave them the feeling of being placed into speakers' life stories as opposed being told a story. Similarly, on discovering that you needed to follow the movement of human voices within the soundscape, $\mathrm{P} 9$ noted that experience became more compelling, more game-like, and it allowed the content to be more easily perceived. By adding movement to the sound, spectral presence was emphasized and engagement was enhanced.

Appreciating the Condition of the Site: The immersive audio content drew visitors into a deeper engagement with the structures and conditions of the landscape. It also afforded visitors a deeper appreciation of the conditions of work at Carrie Furnace. P2 remarked that " $[\mathrm{w}]$ hile hearing about jobs there and looking up at the blast furnace, I realized how scary and intense it would have been to work there." The spatialized soundscape enhanced this immersive quality and allowed visitors to appreciate subtle and overt connections between the virtual sonic content and visual condition of the site. P3 noted the experience of the stationary car area. Walking into the space, they heard past workers recount their experiences while the sounds of hoarding train operating was spatialized and reverberated in the background. P3 shared how the audio enlivened the space, evoking history within the context of the present space: " $[\mathrm{H}]$ earing the sounds of the furnace operating. It made me think much more about the history of the space... hearing trains going by in the background tied the whole experience to the present and the ways in which the history has shaped the city in which we live now." This prompted them to reflect on how they were still "living in this infrastructure."

Affect of Active Sound Elements: Although the structures were static, active sound elements brought participants into a landscape 'living' with its history. As participants matched details from the digital audio with the physical structures, it helped to conjure immersive imagery in their mind and enhance the presence of these elements. P5 recalled "standing underneath the six stoves, which are disproportionately tall, peculiar looking and alienating. Enveloped by the roaring engine sounds, these industrial machines felt alive again." Similar to [40], at times the soundscape evoked visceral, full-body responses. P9 had a "frightening" experienced inside the stocking house 'tunnel'- a dim and enclosed environment. As they walked inside and heard the loud sounds of the material dropping, they felt as if something might really drop on them: "I started acting differently. I want to cover my head to avoid iron drop off." Similarly P4 and P7 experienced that "the object dropping sound in the tunnel, really makes it immersive (P4)." and "The environment was pretty quiet so that the sound dominated your feeling (P7)." In contrast, the Carrie Deer sculpture offered participants respite from the harsh industrial soundscape. P6 remarked "I was alone, listening to artists talking about this place, the sunlight shed through the factory, abandoned factory and art, train passing away in the background, old chairs and table. The nostalgic feeling was hard to express." In both cases, the soundscape stimulates a full immersion in the legacy and condition of the space which goes beyond what either the sonic and visual experience could achieve alone.

Moving Strategies: Within the experience each participant had agency to choose their own path to explore the soundscape. Figure 1 depicts the variety of different routes taken. Common strategies for traversing the site (and soundscape) include randomly wandering, moving towards and along structures of interest, and following other participants. The participants' strategies for walking the landscape at Carrie Furnace are very similar to natural and expected ways walking any landscape. While the MR technology layers on a curated auditory experience, it appears not to interrupt the nature of typical landscape walks. The spatial groupings or other organizational strategies for audio content did not prioritize a particular route or order of site experience; they did not funnel participants towards any discernible standard approach. Equally, participants reported enjoying the experience of walking with the audio content - e.g. P1 "liked the different ambient/industrial sounds and how they moved with you" - as well as being immersed in and engaged with the content. This suggests that participants found a coherent narrative experience regardless of how they traversed the site and the differing temporal, spatial, and narrative experience that resulted.

Limitations: In the current implementation, performance depended on GPS accuracy and could be less stable in areas with higher levels of spatial complexity e.g. varied topologies, constrained indoor spaces. More precise data, including the specifics of users' trajectories and sounds encountered, could be added to better understand the individual and collective experience of our MR approach. Relatedly, we note our evaluation only had eleven participants as it was conducted just prior to the COVID-19 pandemic. An increased 
number of participants would allow deeper evaluation of the project and richer understand perceptions of spectrality and the experience.

\section{CONCLUSIONS}

This work introduces a spatiotemporal mobile mixed-reality audio walk that draws on Derrida's concept of spectrality to haunt a landscape with its own history. This site-specific work is structured as an open-ended and unguided audio walk through content curation, soundscape composition, iterative prototyping and interaction design. The resulting system gives audiences agency to explore the landscape in an open-ended interaction and encounter adaptive narratives about a post-industrial site. A preliminary evaluation with eleven participants provide valuable insights about design approaches to virtual spatialized soundscape and its relationship to the physical landscape. More importantly, by exploring the design opportunities within the relationship between real and virtual landscapes, this project exemplifies how to use sound as medium to evoke spectral memories. This is an initial exploration of spectrality in audio-based MR. We envision many future directions and encourage exploration of Derrida's concept in future technology-mediated cultural heritage experiences.

\section{ACKNOWLEDGMENTS}

We thank Rivers of Steel for their support of this work without which it would not be possible. We would also like to thank our participants, our reviewers, and Daniel Cardoso Llach for their time and generous feedback.

\section{REFERENCES}

[1] Paul M. Aoki, Rebecca E. Grinter, Amy Hurst, Margaret H. Szymanski, James D Thornton, and Allison Woodruff. 2002. Sotto Voce: Exploring the Interplay of Conversation and Mobile Audio Spaces. In Proceedings of the SIGCHI Conference on Human Factors in Computing Systems (CHI '02). Association for Computing Machinery, New York, NY, USA, 431-438. https://doi.org/10.1145/503376.503454 event-place: Minneapolis, Minnesota, USA.

[2] Frauke Behrendt. 2015. Locative Media as Sonic Interaction Design Walking through Placed Sounds. Wi fournal of Mobile Media 9, 2 (2015). https://research.brighton.ac.uk/en/publications/locative-media-as-sonicinteraction-design-walking-through-placed

[3] Steve Benford, Gabriella Giannachi, Boriana Koleva, and Tom Rodden. 2009. From Interaction to Trajectories: Designing Coherent Journeys through User Experiences. In Proceedings of the SIGCHI Conference on Human Factors in Computing Systems (CHI '09). Association for Computing Machinery, New York, NY, USA, 709-718. https://doi.org/10.1145/1518701.1518812 event-place: Boston, MA USA.

[4] Steve Benford, Chris Greenhalgh, Andy Crabtree, Martin Flintham, Brendan Walker, Joe Marshall, Boriana Koleva, Stefan Rennick Egglestone, Gabriella Giannachi, Matt Adams, Nick Tandavanitj, and Ju Row Farr. 2013. Performance-Led Research in the Wild. ACM Transactions on Computer-Human Interaction 20, 3 (July 2013), 14:1-14:22. https://doi.org/10.1145/2491500.2491502

[5] Rose Biggin. 2017. Immersive Theatre and Audience Experience: Space, Game and Story in the Work of Punchdrunk. Springer. Google-Books-ID: 6YM0DwAAOBAJ.

[6] Maria del Pilar Blanco and Esther Peeren. 2013. The Spectralities Reader: Ghosts and Haunting in Contemporary Cultural Theory. Bloomsbury Publishing USA. Google-Books-ID: VYTFAgAAQBAJ.

[7] Elizabeth Boling. 2014. "Making Alcatraz Amazing": The Alcatraz Cellhouse Tour. International Journal of Designs for Learning 5, 2 (Nov. 2014). https: //doi.org/10.14434/ijdl.v5i2.13141 Number: 2.

[8] Ellen Braae. 2015. Beauty Redeemed: Recycling Post-industrial Landscapes. IKAROS Press. Google-Books-ID: kGrerQEACAAJ.

[9] Simon Bradley. 2012. History to go: oral history, audiowalks and mobile media. Oral History (2012), 99-110.

[10] Toby Butler. 2007. Memoryscape: How audio walks can deepen our sense of place by integrating art, oral history and cultural geography. Geography Compass 1, 3 (2007), 360-372.
[11] Janet Cardiff. 2006. Jena Walk (Memory Field). https://cardiffmiller.com/walks/ jena-walk-memory-field/

[12] Alan Chamberlain, Leif Oppermann, Martin Flintham, Steve Benford, Peter Tolmie, Matt Adams, Ju Row Farr, Nick Tandavanitj, Joe Marshall, and Tom Rodden. 2011. Locating experience: touring a pervasive performance. Personal and Ubiquitous Computing 15, 7 (June 2011), 717. https://doi.org/10.1007/s00779010-0351-3

[13] Luigina Ciolfi and Daniela Petrelli. 2016. Walking and designing with cultural heritage volunteers. Interactions 23, 1 (Dec. 2016), 46-51. https://doi.org/10. $1145 / 2848979$

[14] British Broadcasting Corporation. [n.d.]. BBC sound effects library. http: //bbcsfx.acropolis.org.uk/

[15] Jacques Derrida. 1982. Margins of Philosophy. University of Chicago Press. Google-Books-ID: sHYoqEcbgs4C.

[16] Jacques Derrida. 1994. Specters of Marx: The State of the Debt, the Work of Mourning, and the New International. Psychology Press. Google-Books-ID: sEENbAP5FZsC.

[17] Steven Dow, Jaemin Lee, Christopher Oezbek, Blair Maclntyre, Jay David Bolter, and Maribeth Gandy. 2005. Exploring spatial narratives and mixed reality experiences in Oakland Cemetery. In Proceedings of the 2005 ACM SIGCHI International Conference on Advances in computer entertainment technology (ACE '05). Association for Computing Machinery, New York, NY, USA, 51-60. https://doi.org/10.1145/1178477.1178484

[18] Chris Eaket. 2008. Project [Murmur] and the Performativity of Space. Theatre Research in Canada 29 (March 2008). https://doi.org/10.3138/tric.29.1.29

[19] Cally Gatehouse. 2020. A Hauntology of Participatory Speculation. In Proceedings of the 16th Participatory Design Conference 2020 - Participation(s) Otherwise Volume 1 (PDC '20). Association for Computing Machinery, New York, NY, USA, 116-125. https://doi.org/10.1145/3385010.3385024

[20] Owen Gottlieb. 2015. Jewish games for learning: Renewing heritage traditions in the digital age. (2015).

[21] Daniela Hahn. 2014. Performing Public Spaces, Staging Collective Memory: 50 Kilometres of Files by Rimini Protokoll. TDR/The Drama Review 58, 3 (Sept. 2014), 27-38. https://doi.org/10.1162/DRAM_a_00371

[22] Lissa Holloway-Attaway and Lars Vipsjö. 2020. Using Augmented Reality, Gaming Technologies, and Transmedial Storytelling to Develop and Co-design Local Cultural Heritage Experiences. In Visual Computing for Cultural Heritage. Springer, 177-204.

[23] Tim Ingold and Jo Lee Vergunst. 2008. Ways of Walking: Ethnography and Practice on Foot. Ashgate Publishing, Ltd.

[24] Jonathan Joseph. 2001. Derrida's spectres of ideology. Fournal of Political Ideologies 6 (2001), 95-115. https://doi.org/10.1080/13569310120040177

[25] David S. Kirk, Abigail C. Durrant, Jim Kosem, and Stuart Reeves. 2018. Spomenik: resurrecting voices in the woods. Design Issues 34, 1 (Jan. 2018). https://doi.org/ 10.1162/DESI a 00477 Publisher: Massachusetts Institute of Technology Press.

[26] Pontus Larsson, Aleksander Väljamäe, Daniel Västfjäll, Ana Tajadura-Jiménez, and Mendel Kleiner. 2010. Auditory-Induced Presence in Mixed Reality Environments and Related Technology. In The Engineering of Mixed Reality Systems, Human-Computer Interaction Series. ISBN 978-1-84882-734-9. Springer-Verlag London, 2010, p. 143. 143-163. https://doi.org/10.1007/978-1-84882-733-2_8

[27] Jack M Loomis. 2001. Auditory distance perception in real, virtual, and mixed environments. Mixed reality: Merging real and virtual worlds (2001), 201-214.

[28] Nicholas Mariette. 2013. Human Factors Research in Audio Augmented Reality. In Human Factors in Augmented Reality Environments, Weidong Huang, Leila Alem, and Mark A. Livingston (Eds.). Springer, New York, NY, 11-32. https: //doi.org/10.1007/978-1-4614-4205-9_2

[29] David Martin. 2000. Audio guides. Museum Practice 5, 1 (2000), 71-81.

[30] Bryan J McCann. 2019. Live, virtual, and spectral: being present at the prison (tour). Text and Performance Quarterly 39, 2 (2019), 95-115.

[31] Derek P McCormack. 2010. Remotely sensing affective afterlives: The spectral geographies of material remains. Annals of the Association of American Geographers 100, 3 (2010), 640-654.

[32] Rivers of Steel. 2006. National Historic Landmark Nomination: Carrie Blast Furnaces Number 6 and 7. https://riversofsteel.com/_uploads/files/ CarrieLandmarkNominationFinal.pdf

[33] Rivers of Steel. 2019. Our Story. https://riversofsteel.com/about/our-story/

[34] National Park Service U.S. Department of the Interior Northeast Region Philadelphia Support Office. 2002. BATTLE OF HOMESTEAD AND CARRIE FURNACES 6 AND 7. https://npgallery.nps.gov/GetAsset/9f4411b3-a0f0-4654-b7b4$7 \mathrm{ecb} 7791674 \mathrm{~d}$

[35] Karen O'Rourke. 2013. Walking and Mapping: Artists as Cartographers. MIT Press. Google-Books-ID: wI_MIDWcPswC.

[36] Steve Poole. 2018. Ghosts in the Garden: locative gameplay and historical interpretation from below. International fournal of Heritage Studies 24, 3 (2018), 300-314.

[37] Matthew Potteiger and Jamie Purinton. 1998. Landscape Narratives: Design Practices for Telling Stories. John Wiley \& Sons. Google-Books-ID: Z_MunOqKJ_UC.

[38] Rimini Protokoll. 2013. Remote X. https://www.rimini-protokoll.de/website/en/ project/remote-x 
[39] Kyle Rector, Keith Salmon, Dan Thornton, Neel Joshi, and Meredith Ringel Morris. 2017. Eyes-Free Art: Exploring Proxemic Audio Interfaces For Blind and Low Vision Art Engagement. Proc. ACM Interact. Mob. Wearable Ubiquitous Technol. 1, 3 (Sept. 2017). https://doi.org/10.1145/3130958 Place: New York, NY, USA Publisher: Association for Computing Machinery.

[40] Josephine Reid, Erik Geelhoed, Richard Hull, Kirsten Cater, and Ben Clayton. 2005 Parallel worlds: immersion in location-based experiences. In CHI'05 extended abstracts on Human factors in computing systems. 1733-1736.

[41] Blast Theory. 2015. Too Much Information. https:/www.blasttheory.co.uk/ projects/too-much-information

[42] Philipp Wacker, Kerstin Kreutz, Florian Heller, and Jan Borchers. 2016. Maps and location: Acceptance of modern interaction techniques for audio guides. In
Proceedings of the 2016 CHI Conference on Human Factors in Computing Systems. 1067-1071.

[43] Ron Wakkary and Marek Hatala. 2006. Ec(h)o: Situated Play in a Tangible and Audio Museum Guide. In Proceedings of the 6th Conference on Designing Interactive Systems (DIS '06). Association for Computing Machinery, New York, NY, USA, 281-290. https://doi.org/10.1145/1142405.1142448 event-place: University Park, PA, USA.

[44] Jonathan Westin, Anna Foka, and Adam Chapman. 2018. Humanising places: exposing histories of the disenfranchised through augmented reality. International Journal of Heritage Studies 24, 3 (2018), 283-286.

[45] John Wylie. 2007. The spectral geographies of W.G. Sebald. Cultural Geographies - CULT GEOGR 14 (2007), 171-188. https://doi.org/10.1177/1474474007075353 